\title{
Peripartum Cardiomyopathy: A Case Report
}

\section{Ozlem E. BASARAN, E. Seda GUVENDAG GUVEN, Suleyman GUVEN}

Trabzon, Turkey

\begin{abstract}
Peripartum cardiomyopathy is one of the potentially life-threatening complications of pregnancy. It is characterized by symptoms of heart failure in the last months of gestation or within five months after birth. The objective of this case report was to report a case of peripartum cardiomyopathy.
\end{abstract}

A 20-years-old (G1P0) pregnant woman was referred for further evaluation of heart failure in pregnancy. The patient's family and medical histories were both unremarkable. She had a 36 weeks and 2 days dichorionic diamniotic twin pregnancy. Emergency cesarean section was performed under spinal anesthesia. During the early postoperative period, the patient complaint of respiratory distress and the diagnosis of peripartum cardiomyopathy was confirmed with physical examination, laboratory and echocardiography findings. She was transferred to the cardiac intensive care unit and received supplemental oxygen, noninvasive ventilation and intravenous infusion of lidocaine. She well tailored the medical therapy and she and her babies were discharged uneventfully after 10 days of delivery.

The clinicians should keep in mind the diagnosis of peripartum cardiomyopathy in young woman with twin pregnancy in third trimester who presents with symptoms of heart failure even after delivery. Emergent cardiologic evaluation and intervention must be done for this rare and potentially morbid and mortal condition.

Keywords: Ejection fraction, Peripartum cardiomyopathy, Pregnancy

Gynecol Obstet Reprod Med 2020;26(2):146-147)

\section{Introduction}

Peripartum cardiomyopathy (PPCM) is one of the potentially life-threatening complications of pregnancy. The etiopathogenesis is unknown. Peripartum cardiomyopathy (PPCM) is a rare form of myocardial disease in pregnancy which is characterized by left ventricular systolic dysfunction (1). The left ventricular systolic ejection fraction causes heart failure (2). Peripartum cardiomyopathy has been described by the European Society of Cardiology as a form of dilated car-

${ }^{1}$ Health Sciences University, Etlik Zubeyde Hanim Women's Health Education and Researching Hospital, Gynecologic Oncology Clinic

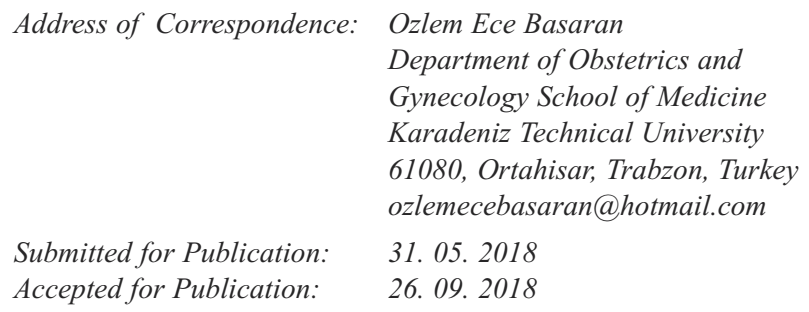

\begin{tabular}{|c|c|}
\hline \multirow{3}{*}{ 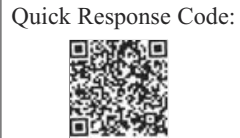 } & Access this article online \\
\hline & $\begin{array}{l}\text { Website: www.gorm.com.tr } \\
\text { e- mail: info@gorm.com.tr }\end{array}$ \\
\hline & DOI:10.21613/GORM.2018-803 \\
\hline
\end{tabular}

How to cite this article: Basaran OE. Guvendag Guven S. Guven S. Peripartum Cardiomyopathy: A Case Report. Gynecol Obstet Reprod Med 2020;26(2):146-147 diomyopathy. The main symptoms are related to heart failure in the last months of gestation or within five months after birth.

\section{Case Report}

A 20-years-old (G1P0) pregnant woman was referred for further evaluation of heart failure in pregnancy. The patient had no history of hypertension, acquired/congenital heart disease, myocarditis, heart valve disease, myocardiopathy or autoimmune disease before pregnancy, and also had no history of drug, cigarette, alcohol or drug use, no known allergy. According to her last menstruation date, she had a dichorionic diamniotic twin pregnancy of 36 weeks and 2 days of gestational age. Pelvic examination revealed $5 \mathrm{~cm}$ cervical dilatation, $90 \%$ of cervical effacement, breech presentation of first fetus. Sonographic examination confirmed dichorionic diamniotic live twin pregnancy with estimated fetal weight of 2.200, and 2.000 gr for each fetus. The presentation of fetuses was breech /vertex. Fetal cardiotocography showed regular uterine contractions.

Following the preoperative cardiology and anesthesia consultations, emergency cesarean delivery was planned because of preterm labor and abnormal fetal presentations. Cesarean section was performed under spinal anesthesia and 2160 gram live female fetus with 7/8 (five and ten minutes) Apgar scores, and 1980 gram live male fetus with 7/8 five and ten minutes) Apgar scores were delivered. During the early postoperative 
period, the patient complaint of respiratory distress. Her general condition was moderate. The patient was pale in appearance and had dyspnea and orthopnea. Pulmonary and cardiac auscultations were normal except tachycardia. Other general physical examination findings were unremarkable.

In the laboratory tests; the hemoglobin was $8.5 \mathrm{~g} / \mathrm{dL}$, the hematocrit was $27.6 \%$, the thrombocyte was $201.000 / \mathrm{mm}^{3}$ and the serum cardiac troponin level was 248.3. Electrocardiography on admission showed sinus tachycardia at 140 beats per minute and negative T waves in V1 and V2. Cardiac ultrasonography showed left ventricular global hypokinesis and dilatation, a prominent pericardial effusion around the right atrium with no signs of tamponade. Subsequently, she was transferred to the cardiac intensive care unit and received supplemental oxygen, noninvasive ventilation and intravenous infusion of lidocaine. During her treatment, she developed non-sustained ventricular tachycardia.

On the third day of admission, her comprehensive echocardiography with biplane Simpson's method revealed an ejection fraction of $50 \%$. On the first day of hospital admission left ventricular ejection fraction was $30 \%$. Based on these findings, the final diagnosis of peripartum cardiomyopathy was made. She well tailored the medical therapy and she and her babies were discharged uneventfully after 10 days of delivery.

Signed patient consent form was obtained for this report.

\section{Discussion}

Although its exact cause is unknown, PPCM is thought to result from increased cardiac stress due to the increased blood volume during pregnancy (1). The incidence in United States is between 1:2.289-1:4.000. The risk factors for PPCM includes multiple pregnancy and higher gestational age. However, $25-75 \%$ of cases occur in young woman giving birth to their first child (2-3). Our patient was 25 years-old and this was her first pregnancy which was also a twin pregnancy.

As other cardiomyopathies, cardiomyopathy in pregnant women without underlying cardiac disease is characterized by the development of systolic dysfunction resulting in cardiac decline of left ventricular ejection fraction. The clinical diagnostic criteria of the disease were determined by Demakis et al in 1971 like this (4): (a) Heart failure developed during the last month of pregnancy or during the first 5 months of postpartum period. (b) No other causes to explain heart failure. (c) The absence of a heart disease defined before last month of pregnancy. (d) Left ventricular dysfunction with ejaculation fraction reduction in echocardiography. The final diagnosis of PPCM can only be made if the woman fulfills all four criteria $(1,4)$. In our case, the diagnosis of PPCM was carried out because of the absence of a history of heart disease, the occurrence of the disease in the postpartum period, the absence of another cause explaining heart failure, and also the presence of echocardiography findings.
Approximately half of the cases show spontaneous and complete recovery of left ventricular function after pregnancy. However, there may be a more progressive disease pattern that may require intensive treatments and even cardiac transplantation. Early diagnosis and treatment is very important (5-6). We were in need of intensive care, but due to early diagnosis and initiation of medical treatment, the need for intensive care unit was avoided and the patient was medically treated without any morbidity.

The main treatment strategies of peripartum cardiomyopathy should include oxygen supplementation, salt restriction, diuretics, digital and vasodilating agents. Cardiac transplantation is the only option for patients not responding to medical treatment (6).

The clinicians should keep in mind the diagnosis of PPCM in young woman with twin pregnancy in third trimester and having heart failure symptoms even following delivery. Emergent cardiologic evaluation and intervention must be done for this rare and potentially morbid and mortal condition. Early diagnosis would save woman life.

Conflict of Interest Statement

The authors have no conflict of interest and funding to declare. Funding: No funding.

Acknowledgement: None

\section{References}

1. Pearson GD, Veille JC, Rahimtoola S, Hsia J, Oakley CM, Hosenpud JD, et al. Peripartum cardiomyopathy: National Heart, Lung, and Blood Institute and Office of Rare Diseases (National Institutes of Health) workshop recommendations and review. JAMA 2000;1;283(9):1183-8

2. Vanzetto G, Martin A, Bouvaist H, Marlière S, Durand M, Chvanon O. Cardiomyopathie du péripartum: une entité multiple. Presse Médicale 2012;41(6 Pt 1):613-20. doi: 10.1016/j.lpm.2012.03.014. Epub 2012 May 1.

3. Hibbard JU, Lindheimer M, Lang RM. A modified definition for peripartum cardiomyopathy and prognosis based on echocardiography. Obstet Gynecol 1999;94(2):311-6.

4. Demakis JG, Rahimtoola SH. Peripartum cardiomyopathy. Circulation 1971;44(5):964-8

5. Roche-Kelly E, Nelson-Piercy C. Managing cardiovascular disease during pregnancy: best practice to optimize outcomes. Future Cardiol 2014;10(3):421-33. doi: 10.2217/fca.14.21.

6. Loyaga-Rendon RY, Pamboukian SV, Tallaj JA, Acharya D, Cantor R, Starling RC, et al. Outcomes of patients with peripartum cardiomyopathy who received mechanical circulatory support. Data from the Interagency Registry for Mechanically Assisted Circulatory Support. Circ Heart Fail 2014;7(2):300-9. 